\begin{tabular}{l} 
Sharif University of Technology \\
Scientia Iranica \\
SCIENTIA \\
IRANICA \\
Transtions A: Civil Engineering \\
\hline
\end{tabular}

\title{
An anisotropic multi-plane elastic-damage model with axial and shear damage and its application to concrete behavior
}

\author{
S.A. Sadrnejad* and M.R. Hoseinzadeh \\ Department of Civil Engineering, K. N. Toosi University of Technology, Tehran, Iran.
}

Received 13 June 2015; received in revised form 14 March 2016; accepted 18 October 2016

\author{
KEYWORDS \\ Multi-planes; \\ Micro-plane; \\ Elastic-damage model; \\ Nonlinear behavior; \\ Quasi-brittle \\ materials; \\ concrete.
}

\begin{abstract}
The prediction of material response is necessary for the analysis of structure under static or dynamic loading. Mathematical modeling of the nonlinear tri-dimensional mechanical behavior of quasi-brittle materials, like concrete, caused by damaging and plasticity effects is one of the most serious classical challenges we face in the engineering science. Among phenomena of different orientations, the micro-plane models, like multiplanes models, which use a constitutive equation in a vectorial form rather than tensorial form by means of capturing interactions, can serve this goal adequately. This paper presents a simple realistic and robust damage based model in the multi-plane framework accomplished with a few parameters for calibration and suitable for engineering purposes without volumetric-deviatoric split of strain tensor and its problems. This damage formulation has been built on the basis of two types of fundamental damage, namely, axial damage and shear damage, that can essentially occur on each micro-plane and, based on this concept, two new axial and shear damage functions are presented. By comparing the results of the proposed model and experimental data, model verification has been done under different loading/unloading/reloading stress/strain paths.
\end{abstract}

(C) 2017 Sharif University of Technology. All rights reserved.

\section{Introduction}

Concrete is one of the most commonly used construction materials in the fields of civil engineering and passive defense all over the world.

Quasi-brittle materials are neither plastic nor brittle. For these types of materials, especially for concrete that is a heterogeneous material with a highly nonlinear behavior, failure is often caused by the initiation and propagation of micro-cracks [1]. Although

\footnotetext{
*. Corresponding author. Tel.: +982188881128

+982188779474-5 (EXT. 409)

Fax: $+982188779385,+982188779476$

E-mail addresses: sadrnejad@kntu.ac.ir (S.A. Sadrnejad);

mhosseinzadeh@mail.kntu.ac.ir (M.R. Hoseinzadeh)
}

doi: $10.24200 /$ sci.2017.4160 both hardening and softening behaviors can be distinguished under compressive loading, only the softening behavior can be observed under tensile loading. Strain softening is one of the most important aspects of concrete behavior in which after the peak strength, the stress decreases whereas the strain increases due to the propagation and coalescence of micro-cracks, micro-voids, and similar defects. To simulate the crack opening and closing under cyclic loading, the stiffness degradation is another important issue.

On the basis of the studies published in scientific papers related to the constitutive modeling of geo-materials, macroscopic invariant-based models lose some of the important features of material behavior such as orientation dependency. On the other hand, the application of discontinuous models for the daily engineering practices still seems to be ambitious. Based 
on the above argument, it seems to be a rational midway solution to use mesoscopic models. Thus, in the present research, the researchers pay attention to the mesoscopic models (micro-plane or multi-plane models), which are nowadays regarded as powerful tools for numerical simulation of the geo and geo-like materials [2].

Since its introduction in the early 1980s, the micro-plane model for concrete has evolved through a series of progressively improved versions labeled as M1 [3,4], M2 [5], M3 [6,7], M4 [8,9], M5 [10,11], M6 [12], and M7 [13,14].

The micro-plane model to finite strain was generalized in $[15,16]$. There are also more micro-plane model formulations in the literature [17-19].

Micro-plane models have also been successfully developed for other materials such as polycrystalline metals, rock, soil, rigid foam and shape memory alloys, fiber-reinforced concrete and composite laminates, etc. [20].

Some crucial merits of micro-plane models were reviewed in Bazant et al. [8] and Broca and Bazant [21]. A significant point that should be considered is that in micro-plane model, the vertex effect is automatically captured whereas in macro constitutive modeling, this aspect is overlooked [13]. This effect has been experimentally demonstrated; the deformation increments that are parallel to the current loading surface in the stress space are not elastic but inelastic [22].

This vertex effect is attached by great importance when the principal stress and strain directions rotate against the material, which is a typical occurrence, especially under dynamic loads [13]. In view of the intrinsic principles of tensorial macro models, which have one or a few loading surfaces, this effect cannot be captured. Since micro-plane models represent multisurface loading with some vectorial limit surfaces at each micro-plane, this problem is intrinsically overcome [13]. In addition, unlike tensorial form, the strain dependence of the yield limit can be easily taken into consideration in the vectorial component from one generic micro-plane [13].

In this paper, a new multi-laminate damage formulation is established in Section 2. In Section 3, to establish the validity of the proposed concrete material model, verification investigations of analytical results with experimental evidence from the stressstrain response of concrete specimens under different loading conditions are presented. Finally, some salient conclusions are presented in Section 4.

\section{Constitutive equations for the multi-plane elastic-damage model}

The mesoscopic models (known as slip-planes or multilaminate models) were developed on the basis of the static constraint formulation before 1983. In the static constraint approach, the stress tensor is projected on the planes [2]. In 1980, Professor Bazant and his colleagues showed that the static constraint induced unstable localizations of softening into a single microplane, which made it very difficult to generalize the model for post-peak strain-softening damage of quasibrittle materials. The extension to strain-softening damage calls for replacing the static constraint by a kinematic constraint, in which instead of the stress tensor, the strain tensor is projected on the microplanes $[3,4,23]$.

In this paper, in each integration point, the macro-strain tensor is projected on the micro-planes which are tangent on the surface of a unit sphere and, then, by introducing the plane constitutive laws, the micro-stress components are calculated; finally, the macro-stress tensor is identified by transferring every stress vector upon any sampling plane to the global coordinate system and, then, they should be integrated on the surface of the unit sphere. In numerical computations, this integral is approximated according to some of the optimal Gaussian integration formulae for a spherical surface, which replaces the integral by a weighted sum over a finite number of micro-planes [13]. This approximation may be written as follows:

$$
\int_{\Omega} f(x, y, z) d \Omega \approx 4 \pi \sum_{k=1}^{n} w_{k} f_{k}\left(x_{k}, y_{k}, z_{k}\right),
$$

where $\Omega$ is surface of a unit hemisphere centered at the material point equation; $n$ is the number of microplanes; and $w_{k}$ is the weight factor and normalized so that $[4,24]$ :

$$
\sum_{k=1}^{n} w_{k}=1 / 2
$$

The number and position of these points are determined by the numerical integration formulation, which has been chosen for performing the integration of an arbitrary function over the surface of the unit sphere; thus, function $f(x, y, z)$ can be considered as an arbitrary function in the global coordinate system $x-y-z$.

The minimum required micro-planes should be in a way that if obtained strain/stress vectors from strain/stress tensor on micro-planes are transferred to the center of unit sphere, the same initial tensor is retained. For this purpose, at least 21 microplanes should be taken into consideration [13]. It is why in order to enhance the accuracy of numerical integration, 34 micro-planes are applied. In Table 1, direction cosines and weights of the integration points and in Figure 1, their positions on the surface of the unit sphere are shown. It is worth noting that the 
Table 1. Definition of micro-planes.

\begin{tabular}{ccccc}
\hline \multirow{2}{*}{$\begin{array}{c}\text { Plane } \\
\text { number }\end{array}$} & Weight & \multicolumn{3}{c}{ Direct cosines of integration points } \\
\cline { 3 - 5 } & & $\boldsymbol{n}_{\mathbf{1}}$ & $\boldsymbol{n}_{\mathbf{2}}$ & $\boldsymbol{n}_{\mathbf{3}}$ \\
\hline 1 & 0.020277985 & 0.5773503 & 0.5773503 & 0.5773503 \\
2 & 0.020277985 & 0.5773503 & -0.5773503 & 0.5773503 \\
3 & 0.020277985 & -0.5773503 & 0.5773503 & 0.5773503 \\
4 & 0.020277985 & -0.5773503 & -0.5773503 & 0.5773503 \\
5 & 0.058130468 & 0.7071068 & 0.7071068 & 0 \\
6 & 0.058130468 & -0.7071068 & 0.7071068 & 0 \\
7 & 0.030091134 & 0.7071068 & 0 & 0.7071068 \\
8 & 0.030091134 & -0.7071068 & 0 & 0.7071068 \\
9 & 0.030091134 & 0 & -0.7071068 & 0.7071068 \\
10 & 0.030091134 & 0 & 0.7071068 & 0.7071068 \\
11 & 0.038296881 & 1 & 0 & 0 \\
12 & 0.038296881 & 0 & 1 & 0 \\
13 & 0.02930006 & 0 & 0 & 1 \\
14 & 0.019070616 & 0.4082483 & 0.4082483 & 0.8164966 \\
15 & 0.019070616 & 0.4082483 & -0.4082483 & 0.8164966 \\
16 & 0.019070616 & -0.4082483 & 0.4082483 & 0.8164966 \\
17 & 0.019070616 & -0.4082483 & -0.4082483 & 0.8164966 \\
\hline
\end{tabular}

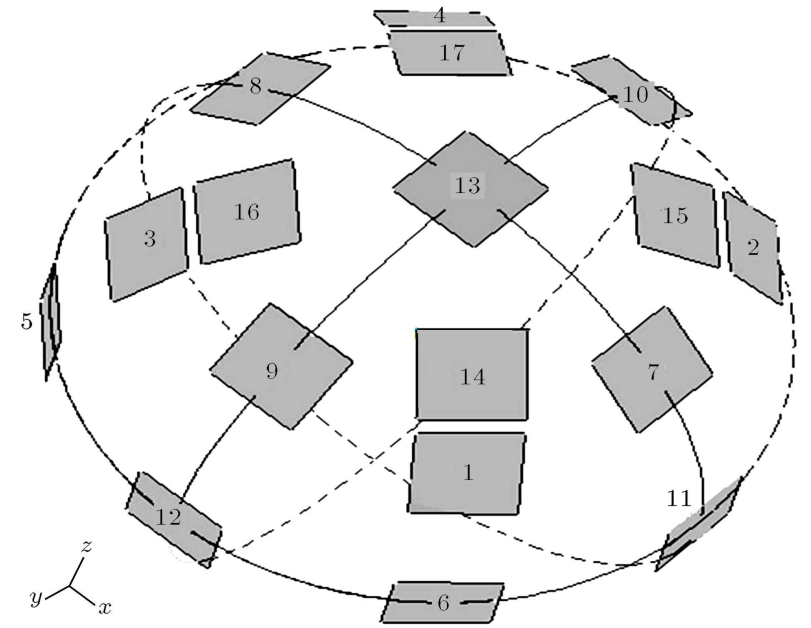

Figure 1. Position of integration points on the unit surface of sphere.

initiation and propagation of all multi-plane models, including micro-plane or multi-laminate models, originate in this mathematical numerical formulation used for integration.

Now, consider the strain tensor in the macro-level state in the center of the unit sphere. This tensor is projected on the planes which are tangent on the surface of the sphere in the prescribed points.

The micro-plane orientation is characterized by the unit normal $n$ of components $n_{i}$, with the subscript $i$ referring to the global Cartesian coordinates $x_{i}(i=$ 1,2 , and 3 ). In the formulation with a kinematic constraint, which makes it possible to describe softening behavior of plain concrete in a stable manner, the

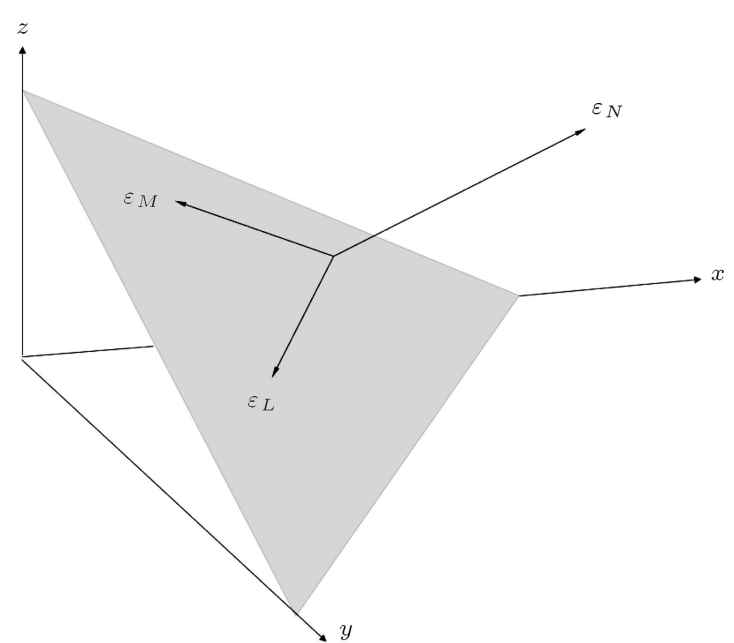

Figure 2. Strain components on a micro-plane.

strain vector $\varepsilon_{N}$ on the micro-plane (Figure 2) is the projection of the macroscopic strain tensor $\varepsilon_{i j}$.

So the components of this vector are $\varepsilon_{N i}=\varepsilon_{i j} n_{j}$. The normal strain on the micro-plane is $\varepsilon_{N}=n_{i} \varepsilon_{N i}$, that is:

$$
\varepsilon_{N}=N_{i j} \varepsilon_{i j}, \quad \quad N_{i j}=n_{i} n_{j} .
$$

The authors also define two coordinate directions $M$ and $L$ in order to characterize the shear strains on the micro-plane (Figure 2), given by two orthogonal units coordinate vectors $m$ and $l$ of components $m_{i}$ and $l_{i}$ lying on the micro-plane. The unit vectors $m$ and $l$ tangential to the plane are considered to be parallel to 
$x-y$ plane so as to minimize directional bias of $m$ and $l$ among micro-planes [13].

The magnitude of the shear strain components on the micro-plane in the direction of $m$ and $l$ is $\varepsilon_{M}=m_{i}\left(\varepsilon_{i j} n_{j}\right)$ and $\varepsilon_{L}=l_{i}\left(\varepsilon_{i j} n_{j}\right)$. Because of the symmetry of tensor, $\varepsilon_{i j}$, the shear strain components may be written as follows:

$$
\varepsilon_{M}=M_{i j} \varepsilon_{i j}, \quad \varepsilon_{L}=L_{i j} \varepsilon_{i j},
$$

in which the following symmetry tensors are introduced:

$$
M_{i j}=\left(m_{i} n_{j}+m_{j} n_{i}\right) / 2, \quad L_{i j}=\left(l_{i} n_{j}+l_{j} n_{i}\right) / 2 .
$$

Once the strain components on each micro-plane are obtained, the stress components $\sigma_{N}, \sigma_{M}$ and $\sigma_{L}$ calculated by defining on-plane constitutive laws between micro-strain and micro-stress vectors through micro-plane, which can be expressed in algebraic or differential forms Eq. (5). The proposed Eq. (5) is used for the simulation of both linear and non-linear behaviors, without splitting vol. and dev. parts, in a way that the theory of elasticity is used for modeling linear elastic behavior while, based on damage mechanics, in order to simulate non-linear aspects, elastic properties of material are reduced in each micro-plane by damage matrix, which is specific to the same micro-plane.

$$
\left\{\begin{array}{c}
\sigma_{N} \\
\sigma_{M} \\
\sigma_{L}
\end{array}\right\}=[D]\left\{\begin{array}{c}
\varepsilon_{N} \\
\varepsilon_{M} \\
\varepsilon_{L}
\end{array}\right\}
$$

in which $[D]$ is the modulus matrix of each micro-plane and is defined according to Eq. (6):

$$
\begin{aligned}
{[D] } & =\left[\begin{array}{ccc}
1-w_{\text {axial }}\left(\varepsilon_{\text {ax }}\right) & 0 & 0 \\
0 & 1-w_{\text {shear }}\left(\varepsilon_{\text {sh }}\right) & 0 \\
0 & 0 & 1-w_{\text {shear }}\left(\varepsilon_{\text {sh }}\right)
\end{array}\right] \\
& \times\left[\begin{array}{ccc}
E_{N} & 0 & 0 \\
0 & E_{T} & 0 \\
0 & 0 & E_{T}
\end{array}\right]
\end{aligned}
$$

The normal and shear stiffness constants, $E_{N}$ and $E_{T}$, on the micro-planes are as follows when the normal micro-plane strains, $\varepsilon_{N}$, are not split into their volumetric and deviatoric parts $[5,25]$ :

$$
\begin{aligned}
& E_{N}=E /(1-2 \vartheta), \\
& E_{T}=E_{N} \cdot(1-4 \vartheta) /(1+\vartheta),
\end{aligned}
$$

where $E$ is Young's modulus on the macro-level; $\vartheta$ is Poisson's ratio; and $E_{N}$ is $K / 3$, where $K$ is the bulk modulus. Only Poisson's ratios in the range of $\vartheta \in$ $[-1,0.25]$ can be reproduced since both $\mathrm{E}_{N}$ and $\mathrm{E}_{T}$ must be nonnegative. This range of $\vartheta$ is sufficient for concrete, for which $\vartheta \approx 0.18$ [13].

$w_{\text {axial }}\left(\varepsilon_{\mathrm{ax}}\right)$ and $w_{\text {shear }}\left(\varepsilon_{\mathrm{sh}}\right)$ in Eq. (6) are the new damage functions. In fact, this damage formulation has been built on the basis of two types of fundamental damage, axial damage, and shear damage, which can essentially occur on each micro-plane. This formulation is vital to describe concrete behavior, because under combined normal and shear damages, cracks tend to fracture in configurations; thus, it is important to explore crack propagation under both mode I (opening) and mode II (sliding) [26].

In this new formulation, according to the damage theory, the values of two new damage functions-that are $w_{\text {axial }}\left(\varepsilon_{\text {ax }}\right)$, axial damage function, and $w_{\text {shear }}\left(\varepsilon_{\mathrm{sh}}\right)$, shear damage function-vary in each micro-plane between zero to one; zero signifies the undamaged state, i.e. no crack initiation on the micro-plane, and one refers to the fully damaged state, i.e. the occurrence of complete crack opening (in mode I) or sliding (in mode II) on the micro-plane. As soon as any crack appears, the stiffness of the micro-plane gradually decreases. The mentioned stiffness component increases again if the on-plane loading conditions change in such a way that crack starts to be closed. As a result, under any arbitrary strain/stress path or change of six strain/stress components, the proposed model is able to predict the concrete behavior. These new damage functions may be written as follows:

$$
\begin{aligned}
& w_{\mathrm{ax}}\left(x\left(\varepsilon_{\mathrm{ax}}\right)\right)=\left[1-\exp \left(-a_{\mathrm{ax}} \times x\left(\varepsilon_{\mathrm{ax}}\right)\right)\right] \cdot H\left(x\left(\varepsilon_{\mathrm{ax}}\right)\right), \\
& w_{\mathrm{sh}}\left(x\left(\varepsilon_{\mathrm{sh}}\right)\right)=\left[1-\exp \left(-a_{\mathrm{sh}} \times x\left(\varepsilon_{\mathrm{sh}}\right)\right)\right] \cdot H\left(x\left(\varepsilon_{\mathrm{sh}}\right)\right) .
\end{aligned}
$$

In the above equations, $H(x(\varepsilon))$ is step function and may be defined as follows:

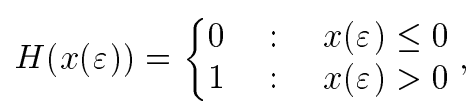

and function $x(\varepsilon)$ is:

$$
x(\varepsilon)=\frac{\varepsilon-\varepsilon_{0}}{\varepsilon_{0}},
$$

in which $\varepsilon$ and $\varepsilon_{0}$ are strain component and damage threshold strain, respectively.

Parameters $a_{\mathrm{ax}}$ and $a_{\mathrm{sh}}$ in Eqs. (8) and (9) are calibrated according to laboratory results and obtained for each specific concrete. $\varepsilon_{\mathrm{ax}}$ is axial strain and $\varepsilon_{\mathrm{sh}}$ is magnitude of shear strain. These strain components may be written as follows:

$$
\begin{aligned}
& \varepsilon_{\mathrm{ax}}=\varepsilon_{N}, \\
& \varepsilon_{\mathrm{sh}}=\sqrt{\varepsilon_{M}^{2}+\varepsilon_{L}^{2}} .
\end{aligned}
$$


Based on Eqs. (8) and (9), the damage functions of each micro-plane are dependent on two key factors; the first one is the parameters $a_{\mathrm{ax}}$ and $a_{\mathrm{sh}}$, which are constant values for all micro-planes in each test, and the other one is the function $x(\varepsilon)$, which has individual values for each micro-plane based on the fact that the magnitude of its strain components is different from those of the other micro-planes. Consequently, the damage functions of each micro-plane are specific to the same micro-plane and are different from those of other micro-planes.

Now, the macro stress tensor from the microstress vectors on the micro-planes can be obtained. To do so, it is first necessary to transfer every stress vector on the micro-plane from local coordinate to the global coordinate system and, then, add up them according to their weightings. In other words, the stress tensor can be calculated as follows:

$$
\sigma_{i j}=6 \sum_{k=1}^{17} w_{k}\left[L_{k}\right]^{T}\left\{\begin{array}{l}
\sigma_{N k} \\
\sigma_{M k} \\
\sigma_{L k}
\end{array}\right\},
$$

in which $[L]$ is the transition matrix of each microplane.

For each micro-plane, the compliance matrix $[C]$ may be defined as follows:

$$
[C]=[D]^{-1} \text {. }
$$

Finally, the macro compliance matrix $[\bar{C}]$ and the macro modulus matrix $[\bar{D}]$ are defined according to Eqs. (15) and (16), respectively:

$$
\begin{aligned}
& {[\bar{C}]=6 \sum_{k=1}^{17} w_{k}\left[L_{k}\right]^{T}\left[C_{k}\right]\left[L_{k}\right],} \\
& {[\bar{D}]=[\bar{C}]^{-1} .}
\end{aligned}
$$

Under cyclic loading, the mechanism of stiffness degradation is more complicated because of the opening and closing of the micro-cracks [27]. The microcracking causing degradation of stiffness occurs in both tension and compression and this degradation becomes more important when the strain increases. Usually, most damage models are not able to reproduce the irreversible strains and the slopes of the curve in unloading and reloading cylices. In order to overcome this problem, often, the plasticity and damage models are combined [27]. In this study, this aspect is simulated by appropriate modifications in the formulation of the proposed micro-plane damage model. To attain this goal, an exponential modification factor as the stiffness recovery factor, $R\left(x\left(\varepsilon_{e q}\right)\right)$, is defined. This factor is multiplied by the stress vector of each micro-plane and may be written as follows:

$$
R\left(x\left(\varepsilon_{e q}\right)\right)=\exp \left(b \times x\left(\varepsilon_{e q}\right)\right),
$$

in which parameter $b$ should be calibrated to give proper cyclic behavior.

In Eq. (17), $\varepsilon_{e q}$ is magnitude of the projected strain vector on each micro-plane defined as follows:

$$
\varepsilon_{e q}=\sqrt{\varepsilon_{N}^{2}+\varepsilon_{M}^{2}+\varepsilon_{L}^{2}} .
$$

In order to split strain tensor into elastic and plastic strain tensors, according to Eq. (19), elastic strain vector is calculated on each micro-plane as follows without classic plasticity law and based upon the assumption that the actual material modulus is higher than their secant modulus.

$$
\begin{aligned}
\left\{\varepsilon^{\text {elastic }}\right\} & =\left\{\begin{array}{l}
\varepsilon_{N}^{\text {elastic }} \\
\varepsilon_{M}^{\text {elastic }} \\
\varepsilon_{L}^{\text {elastic }}
\end{array}\right\} \\
& =\frac{1}{R\left(x\left(\varepsilon_{e q}\right)\right)}\left[\begin{array}{ccc}
E_{N} & 0 & 0 \\
0 & E_{T} & 0 \\
0 & 0 & E_{T}
\end{array}\right]\left\{\begin{array}{c}
\sigma_{N} \\
\sigma_{M} \\
\sigma_{L}
\end{array}\right\} .
\end{aligned}
$$

Plastic strain vector of each micro-plane is obtained by strain vector minus elastic strain vector as follows:

$$
\begin{aligned}
\left\{\varepsilon^{\text {plastic }}\right\} & =\{\varepsilon\}-\left\{\varepsilon^{\text {elastic }}\right\} \\
& =\left\{\begin{array}{c}
\varepsilon_{N} \\
\varepsilon_{M} \\
\varepsilon_{L}
\end{array}\right\}-\left\{\begin{array}{l}
\varepsilon_{N}^{\text {elastic }} \\
\varepsilon_{M}^{\text {elastic }} \\
\varepsilon_{L}^{\text {elastic }}
\end{array}\right\} .
\end{aligned}
$$

According to Eqs. (21) and (22), the elastic and plastic strain tensors are obtained by transferring elastic and plastic strain vectors to the center of the sphere and assembling them with their weighted factors.

$$
\begin{aligned}
& \varepsilon_{i j}^{\text {elastic }}=6 \sum_{k=1}^{17} w_{k}\left[L_{k}\right]^{T}\left\{\varepsilon^{\text {elastic }}\right\}_{k}, \\
& \varepsilon_{i j}^{\text {plastic }}=6 \sum_{k=1}^{17} w_{k}\left[L_{k}\right]^{T}\left\{\varepsilon^{\text {plastic }}\right\}_{k} .
\end{aligned}
$$

Based on the above assumptions, in this paper, as another type of micro-plane models and an improved form of the presented model in [2], the researchers have applied a double-constraint formulation in which the equilibrium and compatibility conditions in every integration point are satisfied simultaneously. This characteristic of the model is called double-constraint. In the proposed model by Labibzadeh and Sadrnejad [2], 11 parameters were used to obtain the results, which were consistent with the experimental results, whereas, in the present model, 3 parameters were applied for this purpose. It should be also added that the reduction in the number of parameters leads to 
a more simplified calibration; this has been done by proposing more elaborate damage functions. Also, in Labibzadeh and Sadrnejad's model [2], 26 micro-planes were used in each integration point and modulus matrix of each plane was multiplied by only scalar damage parameter. In this study, in order to enhance the accuracy of the simulation, all 34 micro-planes are used in each integration point and the modulus matrix of each plane is multiplied by diagonal damage matrix, which includes two parameters of axial damage and shear damage, i.e. axial modulus and shears modulus decrease by the parameters of axial and shear damages, respectively. Therefore, the new proposed model is even able to identify the type of damage dominant in the planes.

As it is obvious, the behavior of many materials depends on orientation, i.e. the stress-strain response of a sample taken from the material in one direction would be different if the sample were taken in a different direction [28]. The term anisotropic is generally applied to describe such behaviors. It is worthwhile to mention that due to the fact that in this model, 34 microplanes have been defined with different orientations in each integration point and each micro-plane behavior is dependent on its three strain components; the final predicted behavior by this model is affected by the behavior in different orientations. In other words, this model has been accompanied by an anisotropy damage mechanics, which is able to predict the concrete behavior under any arbitrary strain/stress path.

\section{Verification studies}

In order to evaluate validity of the proposed concrete material model, verification studies of analytical results with experimental evidence from the stress-strain response of concrete specimens under different loading conditions are presented below. In all figures, the values measured in the tests are shown by circles, and the curves are the model predictions.

In all verifications, the Poisson's ratio is assumed equal to 0.18 .

\subsection{Monotonic loading}

Concrete in compression shows a definite nonlinear behavior with an inelastic irreversible deformation. In the pre-peak stage of concrete response in uniaxial compression, a nonlinear stage is observed, whose amplitude depends on the concrete strength class followed by a softening stage, where brittleness is also dependent on the strength class [29].

As can be seen in Figure 3, there is a good agreement between the results that were obtained by the proposed model and tests data from Van Mier [30] and Karsan and Jirsa [31] under uniaxial compression test.

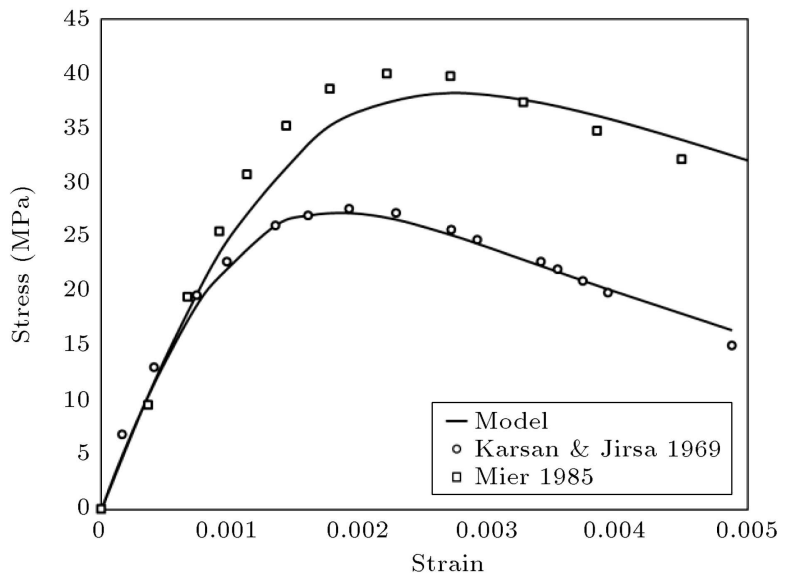

Figure 3. Monotonic compressive uniaxial loading results compared with experimental results.

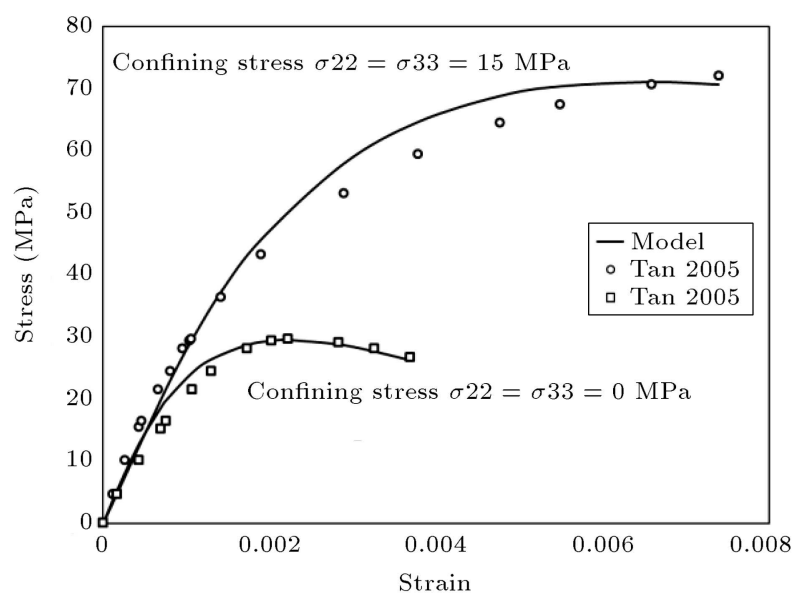

Figure 4. Tri-axial and uniaxial loading results compared with experimental results.

The complexity of concrete behavior increases when submitted to multiaxial stress field that is the current situation of the major Reinforced Concrete (RC) structures. In the Conventional Tri-axial Compression test (CTC test), the hydrostatic pressure is primarily used for the specimen to a certain level and, then, the axial compression is increased while the lateral or confining pressure is held constant. Thus, in these tests, there is not any shear strain on the micro-planes up to the certain level of hydrostatic compression. As shown in Figure 4, there is an admissible agreement between the results that were obtained by the proposed model and by the test data from Tan [32]. Figure 5 depicts the magnitude of axial damage and shear damage on different micro-planes at the end of CTC test.

Experimental tests clearly show that concrete behavior in tension is easily broken and, after the crack appears, concrete begins an increasingly softening behavior with a rotting of tensile strength with the widening of the cracking process. This crack 
opening process is followed by a decrease in crack shear stress transfer because of the deterioration of aggregate interlock. Figure 6 presents uniaxial tension test data from Petersson [33] for two different concretes and their fits by the present model.

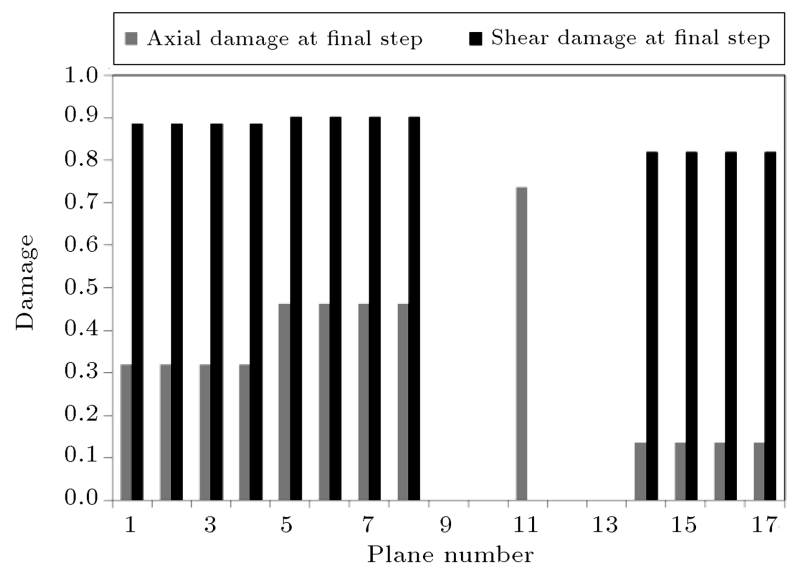

(a)

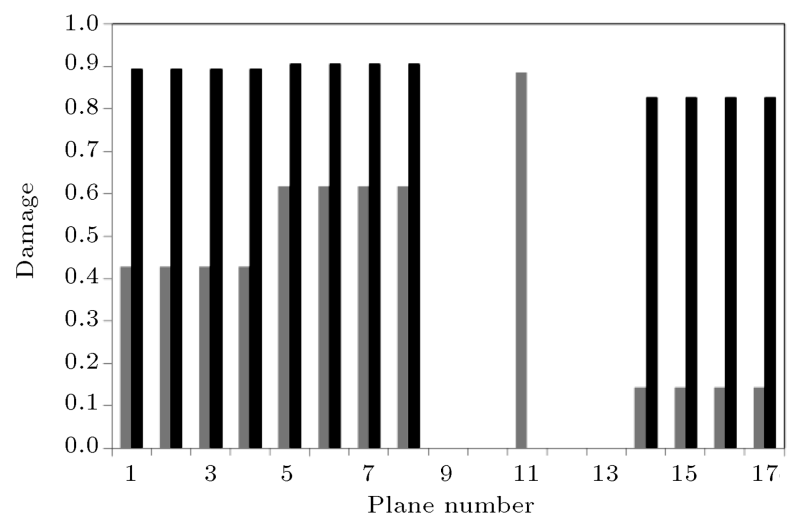

(b)

Figure 5. Magnitudes of axial damage and shear damage on different micro-planes at the end of CTC test: (a) With confinement and (b) without confinement.

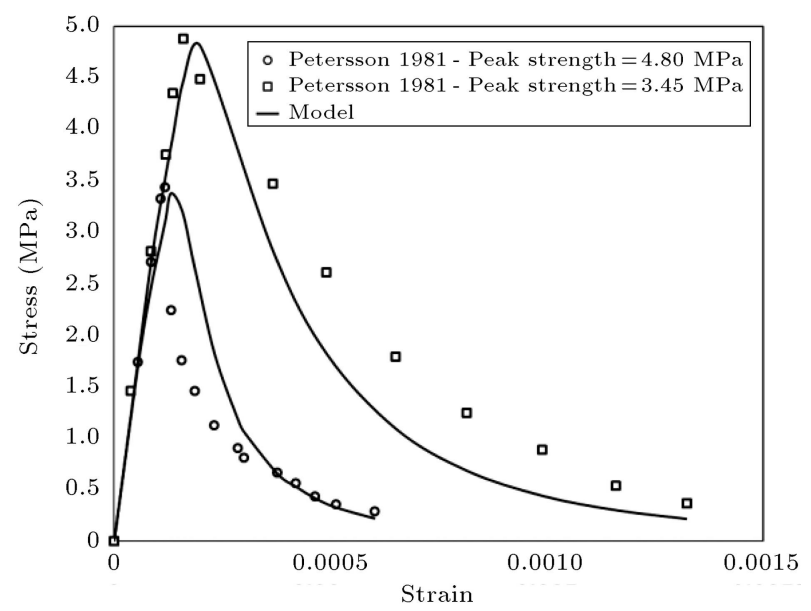

Figure 6. Monotonic tensile uniaxial loading results compared with experimental results.

\subsection{Cyclic loading}

Herein, cyclic loading applications are presented. The main goal is to investigate the capability of the model in capturing stiffness degradation in both tensile and compressive loadings. Figures 7 and 8 illustrate the numerical results from two uniaxial cyclic loading cases, which are compared with the experimental data of Karsan and Jirsa [31] and Gopalaratnam and Shah [34] in each case. In both cases, stiffness degradation has been appropriately simulated at each unloading/reloading cycle. However, it should be highlighted that hysteresis on reloading cannot be captured by the model due to the rate-independent elastic loading/unloading assumption [27].

To show more confidence in the capability of this damage model during the cyclic tests as well as the type and the geometry of damages, Figures 9 and 10 present the variations of damage and stress-strain for both normal and tangential components in the cyclic compression test, respectively.

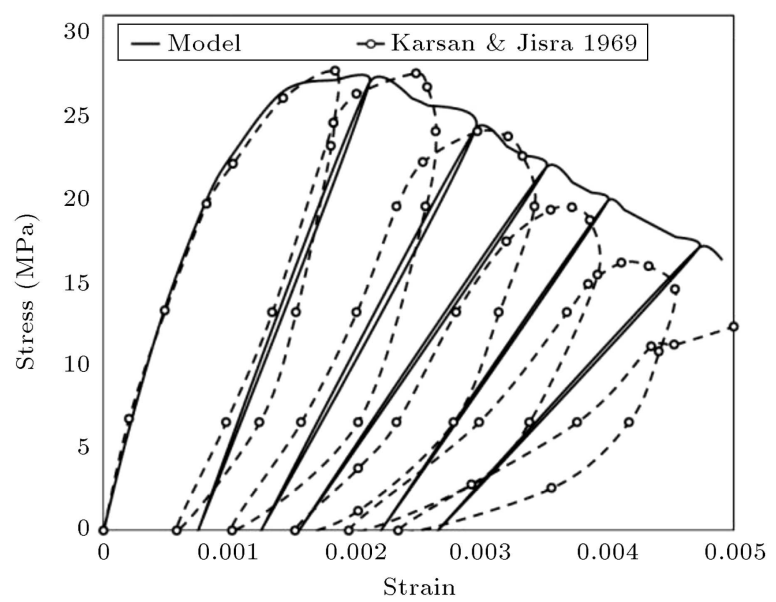

Figure 7. Cyclic compressive uniaxial loading results compared with experimental results.

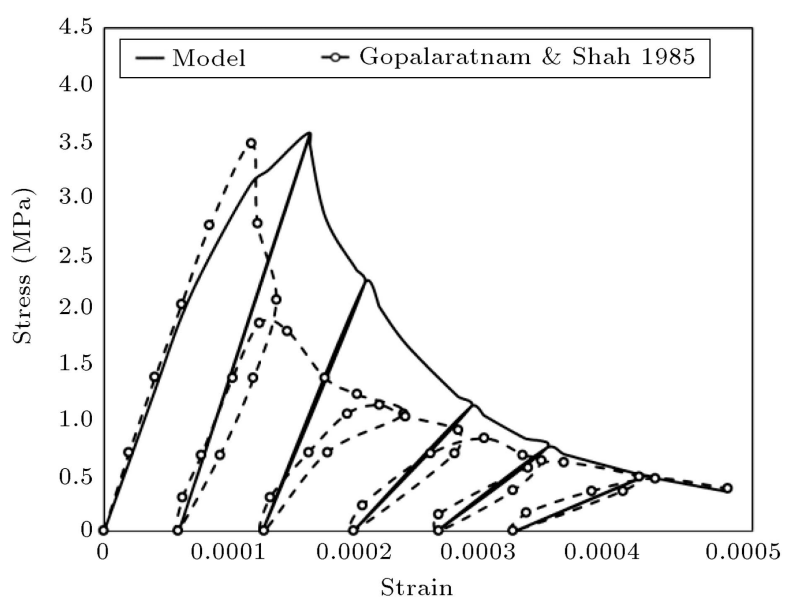

Figure 8. Cyclic tensile loading results compared with experimental results. 


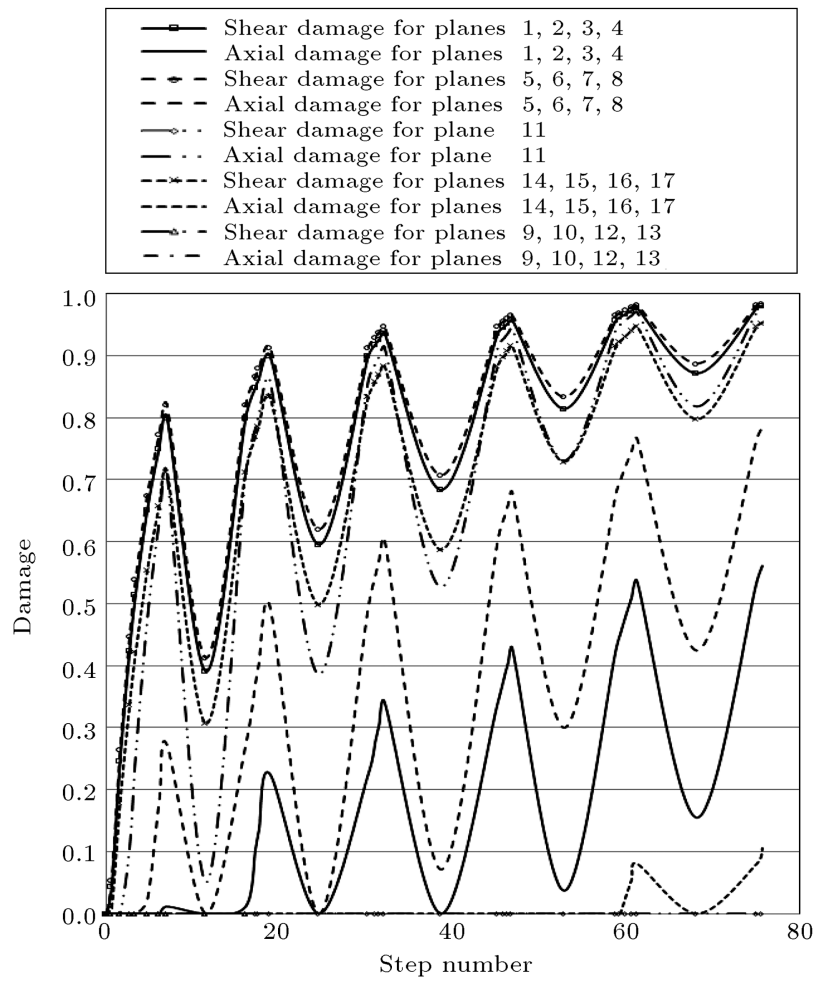

Figure 9. Variations of damage for both normal and tangential components in the cyclic compression test.

Based upon both axial and tangential stress-strain diagrams for each micro-plane, some salient conclusions are listed below.

In view of the effect of the axial and tangential experiences, both axial and shear softening behaviors exist in the micro-planes number 1 to 8 .

Interestingly, in the micro-planes $1,2,3$, and 4 , shear failure is dominant; but, in $5,6,7$, and 8 , both axial and shear failures occur approximately together. However, in the micro-planes 5, 6, 7, and 8, shear failure occurs prior to any other micro-planes failure.

The tangential strain component do not exist in the micro-planes number $9,10,12$, and 13 , and due to their locations, they will under pure tension and remain without any shear damage until the end of loading.

Because of its location, the micro-plane 11 possesses a pure axial strain, which lacks any tangential strain component. The complete degradation of axial stiffness is obviously visible during loading.

In the micro-planes number 14, 15, 16, and 17, under the effect of the experience of axial and shear damages, hardening and softening behaviors are observed respectively; therefore, shear failure is dominant.

\subsection{Parametric study}

There are three free and easily adjustable material parameters which make it possible to simulate compressive or tensile strength, the corresponding strain,
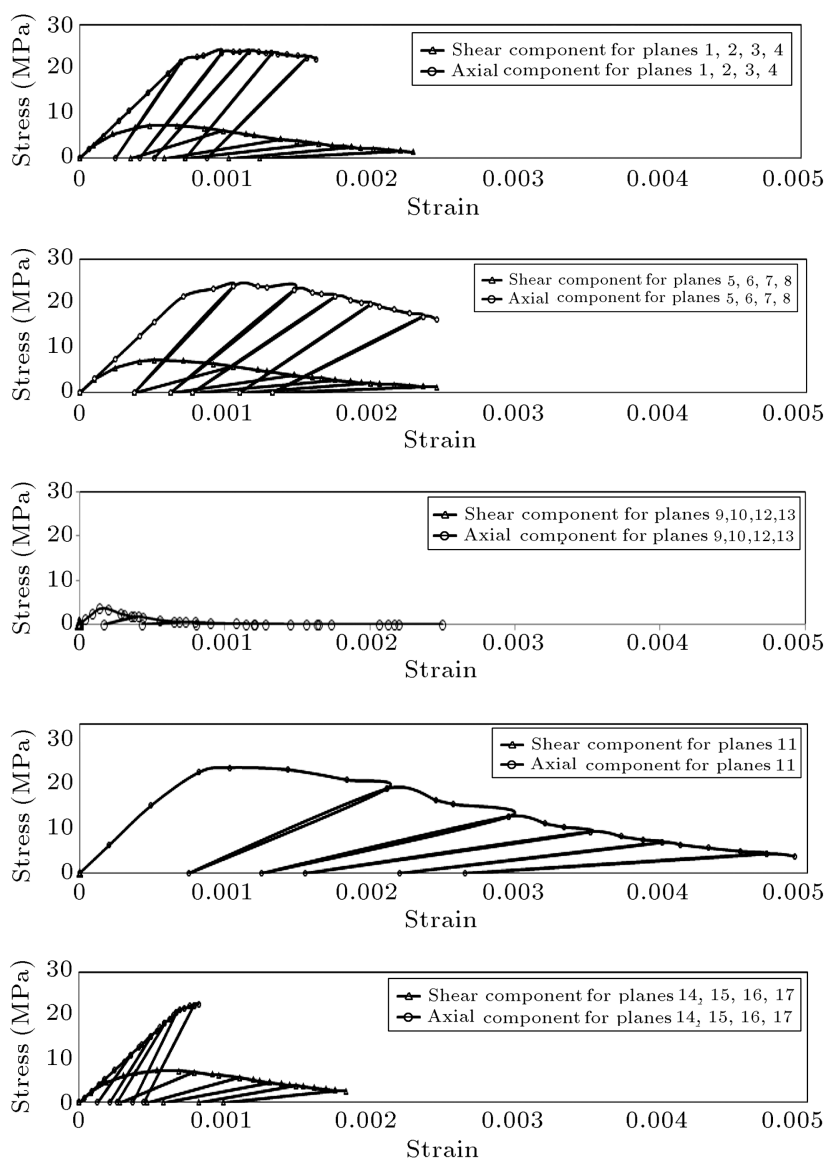

Figure 10. Variations of micro-stress and micro-strain for both normal and tangential components in the cyclic compression test.

the hardening or softening branch, the hydrostatic compression curve, and the cyclic behavior aspects in the proposed model. For each test, the values of the free parameters are listed in Table 2.

\section{Conclusion}

In order to simulate the mechanical behavior of quasi-brittle materials such as concrete under any arbitrary loading, a simple constitutive damage model has been presented using the composition of a new theoretical micro-plane framework and damage approach. This simple model has several features which distinguish it from similar studies that have recently been done in this area. The proposed model is a double-constraint micro-plane, which satisfies both equilibrium and compatibility conditions simultaneously. These damage formulations have been developed on the basis of two types of fundamental damage, axial damage and shear damage, which can essentially occur on each micro-plane. Because of this, the proposed model is able to predict the concrete behavior under any arbitrary strain/stress path or change of six strain/stress components. In this 
Table 2. The values of the free parameters in each test.

\begin{tabular}{cccc}
\hline Test title & \multicolumn{2}{c}{$\begin{array}{c}\text { Monotonic loading } \\
\text { parameters }\end{array}$} & $\begin{array}{c}\text { Cyclic loading } \\
\text { parameter }\end{array}$ \\
\cline { 2 - 4 } & $\boldsymbol{a}_{\text {ax }}$ & $\boldsymbol{a}_{\text {sh }}$ & $\boldsymbol{b}$ \\
\hline Compression test (Mier 1985) & 0.5 & 0.1 & - \\
Compression test (Karsan \& Jirsa 1969) & 0.6 & 0.12 & - \\
Compression test (Tan 2005) & 0.25 & 0.05 & - \\
Tension test (Peterson 1981) & 1.5 & 3 & 0.6 \\
Tension test (Golaparatnam Shah 1985) & 0.95 & 1.9 & 1.5 \\
Cyclic compression test (Karsan \& Jirsa1969) & 0.6 & 0.12 & - \\
Cyclic tension test (Golaparatnam \& Shah 1985) & 1.5 & 3 & - \\
\hline
\end{tabular}

damage model, two new elaborate damage functions are introduced for each micro-plane, in which the axial and shear damage functions are functions of axial strain and shear strain, respectively. To simulate stiffness degradation under cyclic loading, the stiffness recovery factor and the equivalent strain are defined.

These damage evaluation functions are constructed in regard to the experimental evidence on the concrete specimens under compressive and tensile loading conditions reported by the researchers in the literature. In the proposed model, in each phase of numerical calculation, there are two specific damage function values in each micro-plane according to its crack state and, consequently, the stiffness degradation or rehabilitation is evaluated at any physical point of material precisely due to the consideration of crack state data all around it. Thus, by this formulation, according to the damage theory, the value of total damage functions in each micro-plane varies between zero to one; zero is connected with the undamaged state (no crack appears on the micro-plane) and one refers to the fully damaged state (the complete crack opening or sliding occurs on the micro-plane). Moreover, on account of allocation of three micro-plane strain components on each plane in this research, one normal and two others tangent to the plane, it is possible to exactly determine the direction of crack growths around a point. As soon as any crack appears and starts to grow on each of the 34 micro-planes, the stiffness of the micro-plane gradually decreases. The mentioned stiffness component increases again if the on-plane loading conditions change in such a way that crack starts to be closed. Consequently, under any arbitrary strain/stress path or change of six strain/stress components, the proposed model is able to predict the concrete behavior.

This novel micro-plane damage model can simulate the behavior of concrete specimen under both monotonic and cyclic compressive loading and monotonic and cyclic tensile loading with a few model parameter requirements. In this new model, a total of three parameters exist, of which two parameters $a_{\text {ax }}$ and $a_{\text {sh }}$ are always activated and parameter $b$ is activated only under cyclic loading.

The proposed model in this research has several salient characteristics such as pre-failure configuration of the inside material, final failure mechanism identification, and the ability to see induced/inherent anisotropy as well as any fabric effects on material behavior. However, the foundation of this model is logical and simple; thus, it can be effortlessly perceived.

\section{Acknowledgment}

The authors would like to thank anonymous reviewers for their comments, which helped to improve the manuscript.

\section{References}

1. Rasoolan, I., Sadrnejad, S.A. and Bagheri, A.R. "A geometrical inclusion-matrix model for concrete, Int. J. of Civil Eng. 7(2), pp. 73-89 (2009).

2. Labibzadeh, M. and Sadrnejad, S.A. "Mesoscopic damage based model for plane concrete under static and dynamic loadings", Int. J. of Civil Eng.., 4(4), pp. 2011-2019 (2006).

3. Bazant, Z.P. "Micro-plane model for strain-controlled inelastic behavior", Chapter 3, Mech. of Eng. Mat., C. S. Desai and R. H. Gallagher, Eds., Wiley, London, pp. 45-59 (1984).

4. Bazant, Z.P. and Oh, B.H. "Micro-plane model for progressive fracture of concrete and rock", J. Eng. Mech., 111, pp. 559-582 (1985).

5. Bazant, Z.P. and Prat, P.C. "Micro-plane model for brittle plastic material: I. Theory", J. Eng. Mech., 114(10), pp. 1672-1688 (1988a).

6. Bazant, Z.P., Xiang, Y., Adley, M.D., Prat, P.C. and Akers, S.A. "Micro-plane model for concrete: II: Data delocalization and verification", J. Eng. Mech., 122(3), pp. 255-262 (1996b). 
7. Bazant, Z.P., Xiang, Y. and Prat, P.C. "Micro-plane model for concrete. I. Stress-strain boundaries and finite strain", J. Eng. Mech., 122(3), pp. 245-254 (1996a).

8. Bazant, Z.P., Caner, F.C., Carol, I., Adley, M.D. and Akers, S.A. "Micro-plane model M4 for concrete: I. Formulation with work conjugate deviatoric stress", $J$. Eng. Mech., 126(9), pp. 944-953 (2000).

9. Di Luzio, G. "A symmetric over-nonlocal micro-plane model M4 for fracture in concrete", Int. J. Solids Struct., 44(13), pp. 4418-4441 (2007).

10. Bazant, Z.P. and Caner, F.C. "Micro-plane model M5 with kinematic and static constraints for concrete fracture and anelasticity. I. Theory", J. Eng. Mech., 131(1), pp. 31-40 (2005a).

11. Bazant, Z.P. and Caner, F.C. "Micro-plane model M5 with kinematic and static constraints for concrete fracture and anelasticity. II. Computation", J. Eng. Mech., 131(1), pp. 41-47, (2005b).

12. Caner, F.C. and Bazant, Z.P. "Micro-plane model M6f for fiber reinforced concrete", Proc., XI Int. Conf. on Computational Plasticity Fundamentals and Applications, COMPLAS (2011).

13. Caner, F.C. and Bazant, Z.P. "Micro-plane model M7 for plain concrete. I: Formulation", J. Eng. Mech., 139(12), pp. 1714-1723 (2013a).

14. Caner, F.C. and Bazant, Z.P. "Micro-plane model M7 for plain concrete. II: Calibration and verification", $J$. Eng. Mech., 139(12), pp. 1724-1735 (2013b).

15. Bazant, Z.P., Adley, M., Carol, I., et al. "Largestrain generalization of micro-plane model for concrete and application", J. Eng. Mech., 126(9), pp. 971-980 (2000a).

16. Carol, I., Jirsek, M. and Bazant, Z.P. "A framework for micro-plane models at large strain, with application to hyperelasticity", Int. J. Solids Struct., 41(2), pp. 511557 (2004).

17. Kuhl, E. and Ramm, E. "Micro-plane modeling of cohesive frictional materials", Eur. J. Mech. A. Solids, 19, pp. 121-143 (2000).

18. Ozbolt, J., Li, Y. and Kozar, I. "Micro-plane model for concrete with relaxed kinematic constraint", Int. J. Solids Struct., 38(16), pp. 2683-2711 (2001).

19. Leukart, M. and Ramm, E. "Identification and interpretation of micro-plane material laws", J. of Eng. Mech., 132(3), pp. 295-305 (2006).

20. Cusatis, G. and Zhou, X "High-order micro-plane theory for quasi-brittle materials with multiple characteristic lengths", J. of Eng. Mech., 140(7), 04014046 (2014).
21. Brocca, M., Bazant, Z.P. "Micro-plane constitutive model and metal plasticity", Appl. Mech. Rev., 53(10), pp. 265-281 (2000).

22. Caner, F.C., Bazant, Z.P. and Cervenka, J. "Vertex effect in strain-softening concrete at rotating principal axes," J. Eng. Mech., 128(1), pp. 24-33 (2002).

23. Bazant, Z.P. and Oh, B.H. "Micro-plane model for fracture analysis of concrete structures", Proc., Symp. on the Interaction of Non-Nuclear Munitions with Structures, U.S. Air Force Academy, Colorado Springs, CO, pp. 49-53 (1983).

24. Bazant, Z.P. and Oh, B.H. "Efficient numerical integration on the surface of a sphere", Z. Angew. Math. Mech., 66(1), pp. 37-49 (1986).

25. Carol, I and Bazant, Z.P. "Damage and plasticity in micro-plane theory", Int. J. Solids Struct., 34(29), pp. 3807-3835 (1997).

26. Kazemi, M.T. and Vossough Shahvari, F. "Mixed mode fracture of concrete: An experimental investigation", Scientia Iranica, 11(4), pp. 378-385 (2004).

27. Omidi, O. and Lotfi, V. "Numerical analysis of cyclically loaded concrete under large tensile strains by the plastic-damage model", Scientia Iranica, 17(3), pp. 194-208 (2010).

28. H. Sadd, M. "Elasticity", Theory, Applications, and Numerics, 3th Edn., pp. 323-325, Elsevier Press, Oxford, UK (2014).

29. Edalat Behbahani, A., Barros, J.A.O. and VenturaGouveia, A. "Plastic-damage smeared crack model to simulate the behavior of structures made by cement based materials", Int. J. of Solids Struct., 73-74, pp. 20-40 (2015).

30. Van Mier, J.G.M. "Multi-axial strain-softening of concrete: I. fracture; II. Load histories", Mat. and Struct.; 111(19), pp. 179-200 (1986).

31. Karsan, I.D. and Jirsa, J.O. "Behavior of concrete under compressive loading", J. of Struct. Div., ASCE, 95(12), pp. 2535- 2563 (1969).

32. Tan, T.H. "Effects of tri-axial stress on concrete", 30th Conference on Our World in Concrete and Structures, Singapore (2005).

33. Petersson, P.E. "Crack growth and development of fracture zones in plain concrete and similar materials", Rept. TVBM 1006, Lund Institute of Technology, Lund, Sweden (1981).

34. Gopalaratnam, V.S. and Shah, S.P. "Softening response of plain concrete in direct tension", ACI Journal, 3, pp. 310-323 (1985).

\section{Biographies}

Seyed Amirodin Sadrnejad is a Professor in the Department of Civil Engineering at K.N. Toosi University of Technology, Tehran, Iran with over 30 years of professional experience in Civil and Structural Engineering. His research interests cover many different aspects 
of the field and his main expertise and experience are in numerical methods, material modeling, structural mechanics and nonlinear analysis of structures. He has presented at a number of national and international conferences and has published numerous papers in international journals and conferences. He has also authored and co-authored several journal papers in these fields.

Mohamad Reza Hoseinzadeh was born in 1986 and received his BSc degree in 2008 from Shahid Rajaee University, Tehran, Iran with first grade and MSc degree in 2010 from Shahid Chamran University, Ahvaz-Iran, with a GPA of 18.55 out of 20 . Currently, he is a PhD candidate in the Department of Civil Engineering at K.N. Toosi University of Technology, Tehran, Iran. His research interests include material modelling, fracture mechanics, numerical method, nonlinear analysis of structures, and design of seismic and blast resistant structures. 